Historic, archived document

Do not assume content reflects current scientific knowledge, policies, or practices. 

No.1@l4c each-----No.I I-2@llc each----No.2@8c each.

HYBRID TEAS :

Autumn

Ami Quinard

Betty Uprichard

Briarcliff

Caledonia

Condesa de Sastago

Cuba

Dainty Bess

Dame Edith Helen

Duquesa de Panaranda

Edith Nellie Perkins

Editor McFarland

E. G. $\mathrm{Hill}$

Etoile de Hollande

Federico Casas

Golden Dawn

Golden Pernet

Gruss an Teplitz

K. A. Viktoria

Lady Hillingdon

Los Angeles

E. J. Ludding

Luxemburg

Mme. Butterfly

Margaret McGredy

Mevr. G. A. Van Rossem

Mrs. Charies Bell

Mrs. Lovell Swisher

Mrs. P. F. Dupont

McGredy's Scarlet

President Hoover

Radiance

Red Radiance

Roslyn

Seour Therese

Souv.de Mme. C. Chambard

Souv.de Claudius Pernet

Talisman

Willowmere

\section{HYBRID RUGOSAS:}

Burnt orange

Dark Red

Two-tone Pink

Pink

White

Flame

Single Red

Single Pink

Pink

Copper Apricot

Fink

Red

Red

Red

Copper Pink

Yellow

Yellow

Red

White

Yellow

Coral Pink

Fink

Yellowish

Fink \& Gold

Filame

Coppery

Shell Pink

Pink

Yellow

Red

Nulticolored

rink

Ked

Coppery

Yellow

Pink

Yellow

Flame

Fink

Amelie Gravereaux

Belle Pointevine

C. F. Meyer

Dr. Eckener

F. J. Grootendorst

Hansa

Max Graf

Pink Grootendorst

Rugosa Alba

Sarah Van Fleet

Rugosa Agnes

Sir Thomas Lipton

Vanguard

\section{GROOTENDORST SUPREME} Special price

Red
Pink
Pink
Talisman-like
Red
Red
Fink, trailing
Pink
White
Pink
Yellow
White
Orange Salmon

Brilliant Red

\section{HYBRID PERPETUALS:}

Red

White

Red

General Jacqueminot

Henry Nevard

Paul Neyron

Red, everblooming

Pink

\section{PATENTED ROSES:}

Special Prices

American Pride

Black Knight

Dream Parade

Feu Pernet-Ducher

Gloriana

Ireland Hampton

Iuis Brinas

Mme. Cochet-Cochet

Nellie E. Hillock

Sterling

Texas Centennial

Angels Mateu

White

Dark Red

Orange Pink

Yellow

Yellow

Pink

Flame

Coppery Pink

Deep Pink

Pink

Fied Hoover

orange Rose

MISCELIANEOUS :

Austrian Copper

Dr. E. M. Mills

Golden Moss

Harison Yellow

Hugonis

Persian Yellow

Coppery

Primrose Yellow

Golden

Yellow

Yellow

Yellow

\section{POLYANTHAS :}

Chatilion

Clothilde Soupert

Edith Cavell

Else Poulsen

Gloria Mundi

Gruss an Aachen

Kirsten Poulsen

Triomphe d'orleanaise

Popular Pink

Light Pink

Dark Red

Fink

Orange Salmon

Light Pink

Red

Dark Pink

\section{CIIMBERS :}

Belle of Portugal

Clb. Gruss an Teplitz

Dr. W. Van Fleet

Glendale

Jacotte

La Reve

Mary Wallace

Mermaid

Mme. Gregoire Staechelin

(Spanish Beauty)

Paul's Scarlet

Primrose

Roserie

Scorcher

Silver Moon

Fink

Red

Flesh

Yellow

Yellow

Yellow

Luminous Pink

Large Yellow, Single

Fearl Pink

Scarlet

Yellow

Dark Pink

Red

White

\section{SHRUBS :}

Abelia Grandiflora

Amoor River North Privet

Ibolium Privet

Regel Privet

Buddleia alternifolia

Buddleia Ile de France

Ionicera halleana, Hall's Japan

Lonicera heckrotti, Heckrott

Lonicera sempervirens, Red Coral

Orange Quince

Spirea Anthony Waterer, 1 year

Weigelia Rosea

Wisteria Chinensis, 1 year 
